\title{
Planejamento de trajetória, localização e controle aplicados a um quadrirrotor autônomo de corrida
}

\author{
Adriano M. C. Rezende* Henrique N. Machado* \\ Victor R. F. Miranda* Antonio C. B. Chiella* \\ Vinicius M. Gonçalves* Gustavo M. Freitas*
* Programa de Pós-Graduação em Engenharia Elétrica - Universidade Federal de Minas Gerais - Av. Antônio Carlos 6627, 31270-901, Belo
Horizonte, $M G$, Brasil

E-mails: adrianomcr18@gmail.com, henriquebhbr@gmail.com, victormrfm@gmail.com, acbchiella@ufmg.br, mariano@cpdee.ufmg.br, gustavomfreitas@ufmg.br

\begin{abstract}
In this paper, we present a methodology to make an autonomous quadrotor fly through a sequence of gates only with on-board sensors. Our work is a solution to the AlphaPilot Challenge, proposed by the Lookheed Martin Company. In the challenge, the quadriprotor must be able to compete with human-piloted drones in a race. First, we propose a strategy to generate a smooth trajectory that passes through the gates. Then we developed a localization system, which merges image data from an on-board camera with IMU data. Finally, we present an artificial vector field based strategy used to control the quadrotor. Our results are validated with simulations in the official simulator of the competition.

Resumo: Este artigo apresenta uma metodologia para guiar um quadrirrotor autônomo através de uma sequência de portões utilizando apenas sensores onboard. O trabalho é uma solução para o desafio AlphaPilot, proposto pela companhia Lookheed Martin. No desafio, o quadrirrotor deve ser capaz de competir com drones pilotados por humanos em uma corrida. Primeiramente, uma estratégia para gerar uma trajetória suave que trafega através das janelas é proposta. Então, é desenvolvido um sistema de localização que une informações de imagens de uma câmera frontal com informações da IMU. Finalmente, uma estratégia baseada em campos vetoriais artificiais utilizada para controlar o quadrirrotor é apresentada. Os resultados são validados com simulações no simulador oficial da competição.
\end{abstract}

Keywords: Quadrotor race; Perception; Localization; Trajectory planning; Nonlinear control. Palavras-chaves: Corrida de quadrirrotores; Percepção; Localização; Planejamento de trajetória; Controle não-linear.

\section{Introdução}

Nas últimas décadas, os cenários de aplicação da robótica vem crescendo de forma expressiva. Um exemplo é o uso de VANTs (Veículos aéreos não tripulados) nos setores da industria civil e militar, realizando tarefas de forma precisa, ágil e com menor custo. Neste contexto, diversos trabalhos, como em Mahony et al. (2012), vem sendo realizados buscando desenvolver robôs autônomos que possam realizar tarefas de forma similar ou melhor que os humanos.

Neste cenário atual, a empresa norte americana Lockheed Martin $^{1}$, em conjunto com a Drone Racing League ${ }^{2}$ (DRL), lançou um desafio a equipes de pesquisadores do mundo todo, o AlphaPilot Challenge ${ }^{3}$. Este desafio busca o desenvolvimento de quadrirrotores autônomos capazes

\footnotetext{
1 https://lockheedmartin.com/en-us/index.html

2 https://thedroneracingleague.com/

3 https://herox.com/alphapilot
}

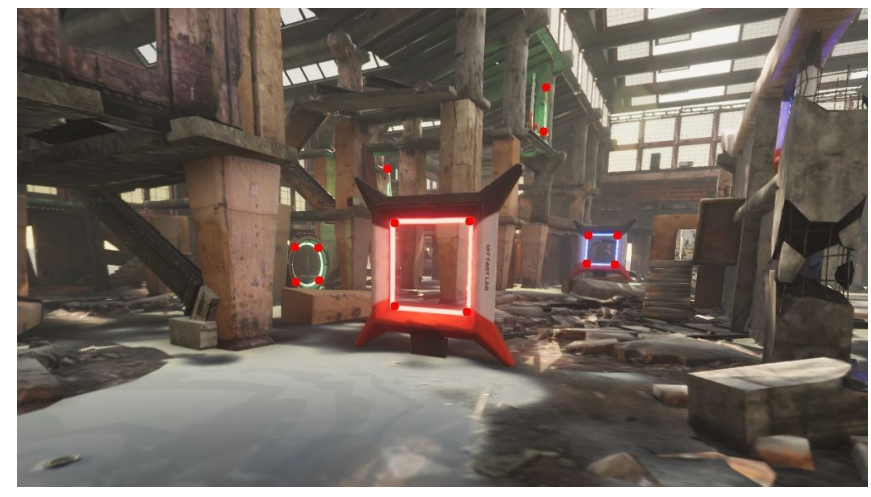

Figura 1. Simulador FlightGoggles. Em foco esta uma janela a ser atravessada pelo quadrirrotor autônomo.

de competir em uma corrida de drones. O grande objetivo é obter um drone autônomo que supere a performance de um pilotado por seres humanos. 
Este trabalho apresenta uma solução para o Teste 3 da competição AlphaPilot. Este teste consiste em fazer um drone passar por uma sequência de janelas que define o trajeto da corrida. O simulador utilizado foi o FlightGoggles, apresentado em Sayre-McCord et al. (2018), desenvolvido por pesquisadores do MIT (Massachusetts Institute of Technology). O presente trabalho consiste na união de diversas técnicas, algumas já bem conhecidas na área de robótica móvel, para a obtenção de uma solução para o desafio. As técnicas estão relacionadas a: (i) planejamento de trajetória; (ii) localização; e (iii) controle.

A Figura 1 apresenta uma imagem do Simulador FlightGoggles, que funciona integrado com o middleware $\mathrm{ROS}^{4}$ facilitando a comunicação entre os sistemas de planejamento, localização e controle. Na figura é possível observar algumas janelas que devem ser atravessadas pelo quadrirrotor no ambiente de simulação.

Diversos métodos de geração trajetórias estão presentes na literatura, cada um com vantagens e e desvantagens. Por exemplo o RRT* (Rapidly Exploring Random Trees), apresentado em Karaman and Frazzoli (2011), tem a grande vantagem de considerar obstáculos no ambiente, mas não gera caminhos suaves, como visto em Machado and Pereira (2018). Outras métodos utilizados na geração de trajetórias são as curvas de Bézier, como em Jayasinghe and Athauda (2016), onde restrições dinâmicas são incluídas no planejamento.

Obstáculos não são considerados neste trabalho, assim características de simplicidade e suavidade motivaram o uso de trajetórias polinomiais. Baseado no trabalho em Mellinger and Kumar (2011), optou-se por trajetórias com baixos valores de snap (segunda derivada da aceleração). Em Mellinger and Kumar (2011) os autores mostram que trajetórias com baixos valores de snap são mais facilmente seguidas pelo quadrirrotor. O método de programação quadrática linear proposto em Richter et al. (2016) foi implementado com esse propósito.

Para seguir uma trajetória o quadrirrotor precisa se localizar no ambiente. O problema de localização é sempre um desafio na robótica móvel. Uma classe de métodos frequentemente utilizados são os baseados em fusão sensorial. Estes métodos buscam uma melhora a estimativa dos estados desejados a partir de dados provenientes de diversos sensores que estejam disponíveis. Tendo em vista a não linearidade típica dos sistemas robóticos, filtros muito utilizados são o Extended Kalman filter (EKF), o Unscented Kalman filter (UKF), e o Filtros de Partículas, que podem ser vistos em Thrun et al. (2005).

Neste trabalho, os sensores utilizados para a localização do drone de corrida considerado são: Inertial Measurement Unit (IMU) com acelerômetro e giroscópio, e uma câmera frontal. Informações de posição relativa entre o drone e uma janela são obtidas a partir da informação da câmera e de um algoritmo PnP (Perspective-n-Point), apresentado, por exemplo, em Gao et al. (2003). Prezando simplicidade e robustez, decidiu-se utilizar um estimador de estados cuja estrutura se assemelha à de um EKF. Este foi baseado em Beard and McLain (2012), onde os autores desenvolvem todos os passos para a construção de um VANT.

\footnotetext{
4 https://www.ros.org
}

Diversos trabalhos também se dedicam ao desenvolvimento de técnicas de controle para quadrirrotores. Por exemplo, em Yousefi and Monfared (2018), os autores utilizam a técnica de Feedback Linearization para o seguimento de trajetória. Em Dolatabadi and Yazdanpanah (2015), os autores usam a técnica de backstepping juntamente com técnicas LQR (Linear-Quadratic Regulator).

Neste trabalho, assume-se que controladores PID atuam no controle de mais baixo nível, que seriam as velocidades dos rotores dada uma velocidade angular desejada para o quadrirrotor. Isso possibilita a exploração de técnicas de controle de mais alto nível. Neste contexto, utilizou-se uma técnica de controle baseada nos campos vetoriais artificiais desenvolvidos em Gonçalves et al. (2010). À técnica de campos vetoriais foi necessário incluir a dinâmica inversa de um modelo do quadrirrotor, técnica análoga à proposta em Rezende et al. (2018) para um VANT de asa fixa.

O restante do artigo é organizado da seguinte forma: na Seção 2 são definidos o desafio e os problemas a serem tratados; na Seção 3 são apresentadas as metodologias para solução dos problemas; na Seção 4 são feitas algumas discussões sobre a solução proposta e alguns detalhes de implementação; na Seção 5 são expostos os resultados obtidos em simulação; finalmente, na Seção 6 são apresentadas as conclusões sobre o trabalho e desenvolvimentos futuros.

\section{Definição do problema}

O desafio proposto na etapa de simulação do Teste 3 do AlphaPilot Challenge consiste em fazer um drone autônomo atravessar uma sequência de janelas no ambiente virtual. A Figura 2 apresenta uma visão aérea do ambiente de simulação. De acordo com a definição do teste, a sequência de janelas que devem ser atravessadas é a indicada na Figura pela linha vermelha e pelas setas em azul.

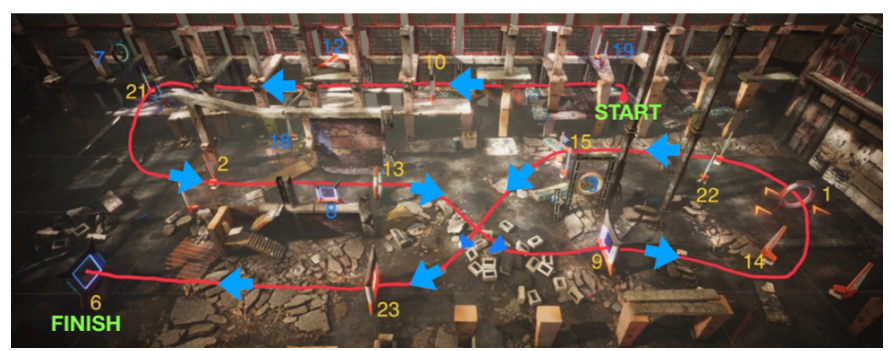

Figura 2. Imagem do simulador FlightGoggles com um exemplo de trajetória a ser percorrida. Fonte: https://www.herox.com/alphapilot/78-test-3

\subsection{Sensoriamento disponível}

Para viabilizar a execução da tarefa, o simulador FlightGogles fornece dados de alguns sensores, a saber: IMU (Inertial Measurement Unit) com acelerômetro e giroscópio, câmera monocular, câmera estéreo e laser de feixe único apontado para baixo. Neste trabalho, apenas a IMU e a câmera monocular foram utilizadas.

O foco do desafio não inclui processamento de imagens. Por esse motivo, o simulador fornece uma informação de quais píxeis da imagem da câmera mono correspondem às projeções dos vértices das janelas. Estes píxeis correspondem aos pontos vermelhos na Figura 1. Estes pontos são os chamados beacons. Além do mais, o simulador fornece 
um identificador para cada beacon, de modo que fica possível identificar a correspondência do píxel com o canto da janela associada. Na prática, estes dados podem ser obtidos de duas formas diferentes: (i) através da fixação de emissores infravermelhos nos vértices das janelas e de uma câmera infravermelho; (ii) através de processamento de imagens RGB.

\subsection{Tarefa}

O desafio consiste em controlar o drone por uma trajetória que passe por uma sequência de $n$ janelas pré-definidas. No desafio AlphaPilot o valor de $n$ foi fixado em 11 janelas, cuja sequência é a apresentada na Figura 2. A solução apresentada neste trabalho, no entanto, pode ser aplicada a qualquer sequência de janelas desejada. Para formalizar a solução do problema, são feitas as seguintes definições:

- $\mathrm{g}^{i} \in \mathbb{R}^{3}$ : posição do centro da $i$-ésima janela, $i=1,2, \ldots, n$, fornecida pelo simulador. Define-se também as componentes de $\mathbf{g}^{i}$ como: $\mathbf{g}^{i}=\left[g_{x}^{i}, g_{y}^{i}, g_{z}^{i}\right]^{T}$;

- $\mathbf{g}^{0} \in \mathbb{R}^{3}$ : posição inicial do drone;

- $\mathbf{v}^{i} \in \mathbb{R}^{3}$ : vetor unitário normal ao plano da $i$-ésima janela. Define-se também $\mathbf{v}^{i}=\left[v_{x}^{i}, v_{y}^{i}, v_{z}^{i}\right]^{T}$;

- $\mathbf{v}^{0} \in \mathbb{R}^{3}$ : vetor nulo, representando a velocidade inicial nula do drone;

- $\mathbf{c}_{j}^{i} \in \mathbb{R}^{3}$ : posição fornecida pelo simulador do $j$-ésimo vértice (beacon), $j=1,2,3,4$, da $i$-ésima janela;

- $\mathbf{b}_{j}^{i} \in \mathbb{R}^{2}$ : coordenadas do $j$-ésimo vértice, $j=1,2,3,4$, da $i$-ésima janela, projetado na imagem atual da câmera frontal do drone.

As posições $\mathbf{g}^{i}$ e $\mathbf{c}_{j}^{i}$, descritas acima são conhecidas, mas com uma faixa de incertezas. Em outras palavras, é conhecida apenas a posição nominal das janelas. Ou seja, as informações fornecidas pelo simulador, $\mathbf{g}^{i}$ e $\mathbf{c}_{j}^{i}$, são apenas uma estimativa da real pose das janelas. As posições reais, que incluem as incertezas nas coordenadas $x$ e $y$ e na rotação em torno do eixo $z$, são desconhecidas a priori.

\subsection{Modelo do quadrirrotor}

O quadrirrotor utilizado no desafio trabalha em um modo de operação Acro, ou seja, as entradas de controle são a força de propulsão (thrust) e as velocidades angulares (referentes aos ângulos de rolagem, arfagem e guinada). Neste trabalho será considerado um modelo não linear com base no proposto em Dolatabadi and Yazdanpanah (2015):

$$
\begin{aligned}
& \ddot{x}=[\cos (\phi) \sin (\theta) \cos (\psi)+\sin (\phi) \sin (\psi)] \frac{u_{1}}{m}-k_{b} \frac{\dot{x}\|\dot{\mathbf{p}}\|}{m}, \\
& \ddot{y}=[\cos (\phi) \sin (\theta) \sin (\psi)-\sin (\phi) \cos (\psi)] \frac{u_{1}}{m}-k_{b} \frac{\dot{y}\|\dot{\mathbf{p}}\|}{m}, \\
& \ddot{z}=-g+\cos (\phi) \cos (\theta) \frac{u_{1}}{m}-k_{b} \frac{\dot{z}\|\dot{\mathbf{p}}\|}{m}, \\
& \dot{\phi}=u_{2}, \\
& \dot{\theta}=u_{3}, \\
& \dot{\psi}=u_{4},
\end{aligned}
$$

onde $x, y$ e $z$ são as coordenadas cartesianas do qua-

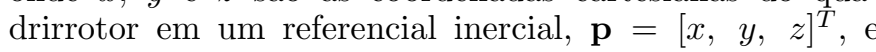
$\phi, \theta$ e $\psi$ são os ângulos de rolagem, arfagem e guinada, respectivamente. A massa do quadrirrotor é dada por $m$, a aceleração da gravidade por $g$ e a constante de atrito viscoso, associada à resistência do ar, é dada por $k_{b}$. As entradas de controle são a força de propulsão $u_{1}$, e as velocidades angulares $u_{2}, u_{3}$ e $u_{4}$.

No simulador FlightGoggles, controladores do tipo PID, já implementados, atuam no controle de baixo nível dos quatro rotores. Tais controladores têm a tarefa de impor ao drone o comando de propulsão e os comandos de velocidades angulares desejados. A alteração da estrutura ou dos ganhos destes controladores não é permitida no desafio.

\section{Solução proposta}

A solução apresentada neste trabalho para o desafio de corrida de drones pode ser dividida em três módulos principais: (i) módulo de planejamento, que planeja uma trajetória a ser seguida dadas as posições nominais das janelas; (ii) módulo de localização, que tem o objetivo de estimar os estados do quadrirrotor, utilizando como sensores a IMU e a câmera frontal para detecção das janelas; (iii) módulo de comando, que calcula sinais de controle que fazem o drone convergir e seguir a trajetória planejada. A Figura 3 ilustra o fluxo de informação entre estes módulos.

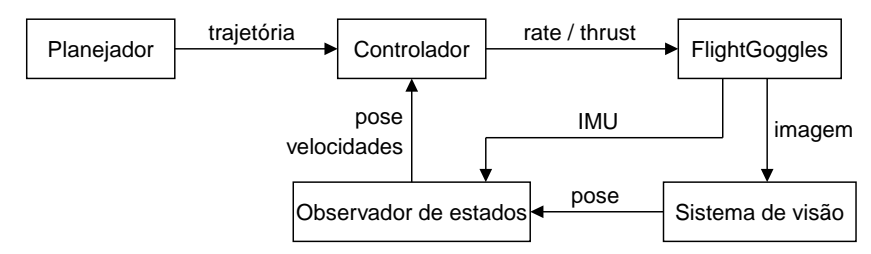

Figura 3. Diagrama de blocos que representa o fluxo de informação entre os módulos da solução proposta.

\subsection{Planejamento de trajetória}

Para cada parte do percurso total a ser executado pelo drone, um segmento de trajetória $\gamma^{i}(\tau) \in \mathbb{R}^{3}$ é gerado, para $i=1,2, \ldots, n$. O primeiro segmento da trajetória completa, $i=1$, conecta o ponto inicial com a primeira janela. Os outros segmentos conectam janelas consecutivas. O objetivo é que a trajetória total esteja de acordo com:

(1) A união dos segmentos de trajetória de cada trecho tem continuidade $C^{1}$;

(2) A trajetória completa atravessa as janelas perpendicularmente aos seus respectivos planos;

(3) a trajetória deve ser tal que o drone fique diante da janela a uma determinada distância mínima dela.

A restrição (1) é interessante porque ela garantirá uma suavidade no chaveamento das trajetórias, o que na prática irá facilitar o trabalho do controlador do drone. A restrição (2) foi determinada com o intuito de maximizar a área da projeção da janela no plano definido pela velocidade do drone, minimizando o risco de colisão. A restrição (3) tem o objetivo de colocar o drone diante da janela em uma determinada distância mínima, de forma que sua câmera possa enxergá-la e permitir assim uma boa localização no momento da travessia.

Com o intuito de calcular cada trajetória, decidiu-se utilizar a minimização do snap como objetivo, devido à facili- 
dade de execução pelo quadrirrotor associada a trajetórias desse tipo. Com esse objetivo, a metodologia proposta em Richter et al. (2016), que considera trajetórias polinomiais, foi implementada. Esta consiste na formulação de um prolema de otimização quadrática, cuja solução pode ser facilmente obtida com a utilização de solvers numéricos.

O algoritmo implementado gera uma trajetória polinomial unidimensional de ordem $N$ entre um instante inicial 0 e um instante final $T$. Para obter uma trajetória em 3 dimensões, isto é, um $\gamma(\tau):[0, T] \rightarrow \mathbb{R}^{3}$, o algoritmo é executado uma vez para cada dimensão $(x, y, z)$. São admitidas restrições de igualdade e de desigualdade para qualquer instante $t \in[0, T]$ no próprio polinômio e em qualquer de suas derivadas até a de ordem $N$. Desta forma, o problema de gerar uma trajetória consiste na definição de um valor para $T$ e de restrições apropriadas.

A posição do ponto de alinhamento associado à restrição (3), seja ela $\mathbf{q}^{i}$, pode ser calculado com base na posição do centro de uma janela $\mathbf{g}^{i}$, no seu vetor normal $\mathbf{v}^{i}$ e em uma distância desejada $d$. Matematicamente temos:

$$
\mathbf{q}^{i}=\mathbf{g}^{i}-d \mathbf{v}^{i}
$$

Uma opção seria requerer que a trajetória passe através do ponto $\mathbf{q}^{i}$. Assim o drone já ficaria diante da janela a uma distância mínima antes de atravessa-la, melhorando sua localização e minimizando as chances de colisão. Para relaxar o problema de otimização, será requerido que a trajetória passe nas proximidades de $\mathbf{q}^{i}$. Na Figura 4 a região em torno do do ponto $\mathbf{q}^{i}$ está representada pelo cubo vermelho. Define-se a aresta do cubo possui $2 \delta_{q} \geq 0$ de largura, $\operatorname{com} \delta_{q}$ definido a priori.

Considere $v_{d} \in \mathbb{R}^{+}$como sendo a velocidade média aproximada que o drone deve executar na trajetória. Por enquanto, a metodologia para a obtenção de um valor para $v_{d}$ consiste em escolher o maior $v_{d}$ de tal forma que o quadrirrotor consiga executar a trajetória. Esta velocidade será utilizada na definição do tempo final $T$, uma vez que este é uma entrada do algoritmo de otimização. Assim, para cada segmento de trajetória o tempo $T^{i}$ é inicialmente estimado com base em uma heurística que leva em conta uma estimativa do comprimento do segmento e a velocidade $v_{d}$. Com esse objetivo, o comprimento $L^{i}$ da $i$-ésima trajetória é previamente estimado como:

$$
L^{i}=\left\|\mathbf{g}^{i-1}-\mathbf{q}^{i}\right\|+\left\|\mathbf{q}^{i}-\mathbf{g}^{i}\right\|,
$$

onde a primeira parcela corresponde à distância da janela anterior (ou do ponto de partida caso $i=1$ ) até o ponto de alinhamento da janela $i$ e a segunda parcela à distância deste ponto à janela. Na Figura 4 esta distância corresponde à soma das distâncias do centro do cubo vermelho (ponto de alinhamento) às duas janelas.

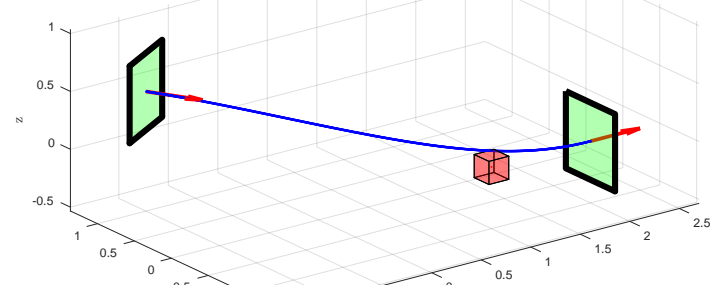

Figura 4. Representação de um segmento de trajetória entre duas janelas consecutivas.
Uma vez calculado $L^{i}$, o intervalo de tempo $T^{i}$ da trajetória $\gamma^{i}$ é definido como:

$$
T^{i}=\frac{L^{i}}{v_{d}}
$$

É também necessário definir o instante em que a trajetória deve passar pelo cubo no entorno do ponto de alinhamento. Este tempo deve ser uma fração $0<\eta^{i}<1$ de $T^{i}$. A estimativa de $\eta^{i}$ é feita com base na razão entre distância até o ponto de alinhamento e a distância total. Assim, define-se:

$$
\eta^{i}=\frac{\left\|\mathbf{g}^{i-1}-\mathbf{q}^{i}\right\|}{L^{i}} .
$$

Finalmente, com os valores de $\mathbf{g}^{i}, \mathbf{v}^{i}, \mathbf{q}^{i}, T^{i}$ e $\eta^{i}$, pode-se escrever problema de otimização para o cálculo da trajetória $\gamma^{i}$. O Modelo 1 apresenta o problema de otimização para o cálculo das componentes em $x$ de cada trecho de trajetória $i=1,2, \ldots, n$. Problemas equivalentes são definidos para as componentes y e $z$. Assim, para o cálculo da trajetória completa, $3 n$ problemas como o apresentado no Modelo 1 são resolvidos.

Modelo 1. (Otimizador de trajetória).

$$
\min _{\alpha_{x}^{i}}: \int_{0}^{T^{i}}\left(\frac{d^{4}}{d \tau^{4}} \gamma_{x}\left(\tau^{\prime}\right)\right)^{2} d \tau^{\prime}
$$

sujeito a:

Casamento de posição:

$$
\begin{aligned}
& \gamma_{x}(0)=g_{x}^{i-1}, \\
& \gamma_{x}\left(T^{i}\right)=g_{x}^{i} .
\end{aligned}
$$

Casamento de velocidade:

$\dot{\gamma}_{x}(0)=v_{d} v_{x}^{i-1}$,

$\dot{\gamma}_{x}\left(T^{i}\right)=v_{d} v_{x}^{i}$.

Passagem pelo cubo de alinhamento:

$$
q_{x}^{i}-\delta_{q} \leq \gamma_{x}^{i}\left(\eta^{i} T^{i}\right) \leq q_{x}^{i}+\delta_{q},
$$

onde $\alpha_{x}^{i}$ é o vetor de $N+1$ coeficientes da trajetória $\gamma_{x}(\tau)$, ou seja as variáveis de otimização.

O funcional de custo no Modelo 1 é a integral do quadrado do snap da trajetória. O primeiro conjunto de restrições faz com que a trajetória comece na janela anterior e termine na janela $i$, fazendo assim o casamento da posição dos segmentos de trajetória consecutivos. O segundo grupo de restrições faz com que as velocidades nas extremidades do segmento de trajetória tenham módulo $v_{d}$ e sejam perpendiculares ao plano da janela associada. Na Figura 4, estas restrições são ilustradas pelos vetores em vermelho. A última restrição, de desigualdade, requer que a trajetória passe nas proximidades do ponto de alinhamento. Esta restrição é equivalente a requerer que no instante $\eta^{i} T^{i}$ a trajetória passe pelo cubo vermelho da Figura 4. A utilização de uma restrição de desigualdade é importante aqui para relaxar a heurística definida por (3), (4) e (5).

Note que a trajetória gerada define valores desejados apenas para a posição $(x, y$ e $z)$ do quadrirrotor. A referência para a guinada será definida na Seção 3.3 de forma que a câmera frontal aponte para a próxima janela.

Finalmente, a trajetória final é dada pela concatenação das trajetórias $\gamma^{i}(\tau)=\left[\gamma_{x}^{i}(\tau), \gamma_{y}^{i}(\tau), \gamma_{z}^{i}(\tau)\right]^{T} \operatorname{com} i=$ $1,2, \ldots, n$. O drone então começa a corrida seguindo a 
trajetória $i$. Quando o sistema de localização detecta que o drone chegou ao fim da trajetória $i$ (travessia da janela i) o chaveamento $i \leftarrow i+1$ é feito e a próxima trajetória começa a ser seguida.

\subsection{Localização}

Nesta seção serão apresentados os métodos utilizados para localizar o drone com base na câmera mono frontal e na IMU. Primeiramente, apresenta-se a metodologia que permite extrair da informação da câmera a pose do drone relativa à uma janela. Em seguida, o estimador de estados que combina esta informação com os dados da IMU é apresentado.

\subsubsection{Medição da pose do drone com respeito ao mundo:} Uma vez que: (i) a posição aproximada $\mathbf{c}_{j}^{i}$ dos cantos das janelas é conhecida; e (ii) as projeções destes vértices no plano da câmera frontal $\mathbf{b}_{j}^{i}$ também são conhecidas; a localização relativa do drone pode ser prontamente calculada com um algoritmo PnP. A Figura 5 representa a projeção de quatro pontos do espaço tridimensional no plano da câmera (retângulo azul). No caso deste trabalho, as bolas cheias na direita equivalem aos cantos de uma janela, enquanto as cruzes no plano equivalem às informações dos beacons, fornecidas pelo simulador. Dado um conjunto de

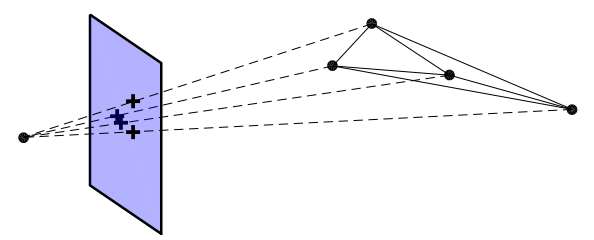

Figura 5. Projeção de pontos no plano de uma câmera.

4 pares de pontos, no caso $\left(\mathbf{c}_{j}^{i}, \mathbf{b}_{j}^{i}\right), j=1,2,3,4$, os algoritmos $\mathrm{PnP}$ retornam uma posição $\mathbf{p}_{m}$ e uma orientação $\mathbf{r}_{m}=\left[\phi_{m}, \theta_{m}, \psi_{m}\right]^{T}$, em relação ao referencial ao qual os pontos $\mathbf{c}_{j}^{i}$ são escritos. Uma estimativa da velocidade $\mathbf{v}_{m}$ do drone no referencial do mundo pode ser obtida derivando-se, numericamente, a medição $\mathbf{p}_{m}$.

Neste trabalho considerou-se a localização relativa à apenas uma janela por vez. Assim, quando o drone está seguindo a $i$-ésima trajetória, a caminho da janela $i$, apenas as informações desta janela são utilizadas na estimativa da pose. O uso apenas da janela $i$ é importante dado que, quando a trajetória corrente é a $i$-ésima, deve-se ter uma localização precisa relativa à janela $i$. Posições relativas às outras janelas, ou até mesmo relativas ao referencial inercial, não são de suma importância neste momento. De fato, uma vez que os valores de $\mathbf{c}_{j}^{i}$ não são as posições exatas dos cantos da janela (veja Seção 2.2), a medição $\mathbf{p}_{m}$ em relação ao referencial inercial carrega o erro presente em $\mathbf{c}_{j}^{i}$. No entanto, a estimativa em relação a janela é apropriada.

Em diversas situações a câmera não detecta os quatro beacons da janela. Um caso comum é quando o drone está em alta velocidade, mantendo o ângulo de arfagem relativamente elevado para a compensação da resistência do ar. Nestas situações, a câmera fica apontada para baixo e frequentemente detecta apenas 2 beacons da janela. No caso em que 3 pontos são detectados, os algoritmos conseguem retornar a medição dada uma estimativa inicial. A saída do filtro a ser descrito adiante foi então utilizada com esse propósito. No caso em que apenas 2 pontos são detectados, dada uma estimativa inicial apenas uma melhora nesta estimativa é retornada.

3.2.2 Estimação dos estados: O sistema de observação de estados tem por objetivo estimar a posição, a orientação e a velocidade do quadrirrotor. A estimativa de posição é dada pelo vetor $\overline{\mathbf{p}}$ com respeito à um referencial inercial. A estimativa de orientação é dada pelo vetor $\overline{\mathbf{r}}=[\bar{\phi}, \bar{\theta}, \bar{\psi}]^{T}$. A estimativa da velocidade, em relação ao corpo do drone, é dada pelo vetor $\overline{\mathbf{v}}_{b}$.

O estimador de estados utilizado foi baseado no observador de estados apresentado em (Beard and McLain, 2012, p. 151). O estimador possui estrutura similar aos estimadores Bayesianos, com uma etapa de predição e outra de atualização. Na etapa de predição, o seguinte modelo é utilizado:

$$
\begin{aligned}
& \dot{\overline{\mathbf{p}}}=R_{b w} \overline{\mathbf{v}}_{b}, \\
& \dot{\overline{\mathbf{r}}}=J_{r} \boldsymbol{\Omega}_{b}, \\
& \dot{\overline{\mathbf{v}}}_{b}=\mathbf{a}_{b}+\boldsymbol{\Omega}_{b} \times \overline{\mathbf{v}}_{b}+g R_{b w}^{T} \hat{\mathbf{k}},
\end{aligned}
$$

onde $R_{b w} \equiv R_{b w}(\overline{\mathbf{r}}) \in S O(3)$ representa a orientação do drone. Os vetores $\boldsymbol{\Omega}_{b}$ e $\mathbf{a}_{b}$ são as medições de velocidades angulares e acelerações lineares respectivamente, ambas no referencial do drone, dadas pelo giroscópio e pelo acelerômetro da IMU. $J_{r} \equiv J_{r}(\bar{\phi}, \bar{\theta})$ é a matriz Jacobiana que relaciona as velocidades angulares no referencial inercial com as velocidades angulares no referencial do corpo. O termo $\boldsymbol{\Omega}_{b} \times \overline{\mathbf{v}}_{b}$ é uma compensação das acelerações de Coriolis, $g$ é a aceleração gravitacional e $\hat{\mathbf{k}}$ é o vetor canônico na direção $z$. Note que o modelo cinemático é a tempo continuo, sendo discretizado através de integração numérica.

Na etapa de atualização (ou correção) as informações provenientes do sistema de percepção são utilizadas para corrigir os valores da estimativa dos estados do filtro. Assim, as medições $\mathbf{p}_{m}, \mathbf{r}_{m}$ e $\mathbf{v}_{m}$ são utilizadas para atualizar os estados $\overline{\mathbf{p}}, \overline{\mathbf{r}}$ e $\overline{\mathbf{v}}_{b}$ respectivamente. A atualização da estimativa de posição é então dada por:

$$
\overline{\mathbf{p}} \leftarrow \overline{\mathbf{p}}+K_{p}\left(\mathbf{p}_{m}-\overline{\mathbf{p}}\right),
$$

onde $0<K_{p}<1$, é um ganho constante. Observe que a estrutura do estimador é bem semelhante à de um filtro Kalman, porém, a incerteza dos estados não são estimadas. Assim como no filtro de Kalman, a diferença entre a medição e a estimativa da medição é a chamada inovação.

Note que, uma vez que a medição $\mathbf{p}_{m}$ é relativa à próxima janela, a estimativa de posição estará correta em relação à próxima janela. Assim, se a janela real está deslocada em relação à posição nominal, a estimativa $\overline{\mathbf{p}}$ admitira o mesmo deslocamento em relação ao referencial inercial. Como interesse é atravessar a janela, a variável de interesse é a pose relativa à janela $i$, que estará sendo apropriadamente estimada.

A estimativa de velocidade é feita de forma análoga à de posição. No entanto, deve-se lembrar que a velocidade medida está no referencial inercial, o que faz necessário o uso da transformação de rotação $R_{b w}$. Temos então:

onde $0<K_{v}<1$.

$$
\overline{\mathbf{v}}_{b} \leftarrow \overline{\mathbf{v}}_{b}+K_{v}\left(R_{b w}^{T} \mathbf{v}_{m}-\overline{\mathbf{v}}_{b}\right)
$$

Diferentemente das etapas de atualização em (7) e em (8), na atualização dos ângulos de orientação foi considerado 
que a inovação é dada pelo seno da diferença entre os ângulos medidos e as estimativas atuais. O intuito aqui é o de evitar problemas com deslocamentos de $2 \pi$ na inovação. Assim, a atualização dos ângulos de Euler é dada por:

$$
\begin{aligned}
& \bar{\phi} \leftarrow \bar{\phi}+K_{r} \sin \left(\phi_{m}-\bar{\phi}\right), \\
& \bar{\theta} \leftarrow \bar{\theta}+K_{r} \sin \left(\theta_{m}-\bar{\theta}\right), \\
& \bar{\psi} \leftarrow \bar{\psi}+K_{r} \sin \left(\psi_{m}-\bar{\psi}\right),
\end{aligned}
$$

onde $0<K_{r}<1$. Note que $\sin (x) \approx x$, então, para pequenos valores de inovação, a utilização do seno pouco interfere na dinâmica do filtro, apenas trata os deslocamentos de $2 \pi$.

\subsection{Controle}

A estratégia adotada para controlar o quadrirrotor ao longo das trajetórias planejadas é baseada em campos vetoriais artificiais. Dado um campo vetorial cujas linhas integrais convergem para curva desejada, leis de controle adicionais são definidas para fazer o modelo em (1) seguir o campo vetorial.

A Figura 6 representa o diagrama de controle utilizado. O campo vetorial fornece, além de um vetor velocidade, a sua Jacobiana, utilizada no controle. O modelo inverso do drone é utilizado para gerar sinais de controle. Os blocos dentro do retângulo tracejado são o controlador PID de baixo nível e o modelo simulado no FlightGoggles, equivalendo então ao modelo em (1).

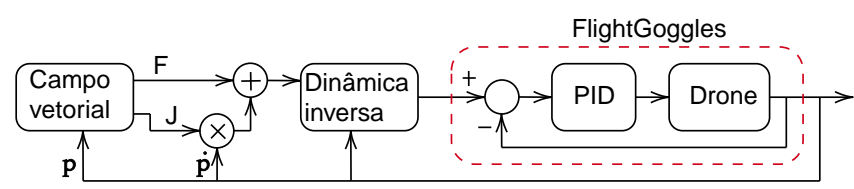

Figura 6. Representação da estrutura do controlador.

3.3.1 Campo vetorial: Seguindo na linha do trabalho em Gonçalves et al. (2010), o campo vetorial aqui utilizado para resolver o problema de controle também consiste na definição de duas componentes, uma tangente e uma de convergência para a curva.

Considere a função $\mathbf{D}: \mathbb{R}^{3} \rightarrow \mathbb{R}^{3}$ definida como sendo o vetor distância entre a posição estimada do drone $\overline{\mathbf{p}}$ e a curva $\gamma^{i}$. Em outras palavras, $\mathbf{D}=\overline{\mathbf{p}}-\gamma^{i}\left(\tau^{*}\right)$, onde $\tau^{*}$ é definido como:

$$
\tau^{*}=\arg \min _{\tau}:\left\|\overline{\mathbf{p}}-\gamma^{i}(\tau)\right\| .
$$

Os vetores unitários de convergência e tangente são definidos como:

$$
F_{\text {conv }}=\frac{\mathbf{D}}{\|\mathbf{D}\|}, \quad F_{\text {tang }}=\frac{d \gamma^{i}\left(\tau^{*}\right)}{d \tau}\left\|\frac{d \gamma^{i}\left(\tau^{*}\right)}{d \tau}\right\|^{-1} .
$$

É possível provar que estes dois vetores são perpendiculares, consequência direta da condição de otimalidade do problema em (10).

Considere agora $D=\|\mathbf{D}\|$. Similarmente ao que foi feito em Gonçalves et al. (2010), dado um peso de convergência $k_{f}>0$ define-se funções $G \equiv G(D)=-(2 / \pi) \operatorname{atan}\left(k_{f} D\right)$ e $H \equiv H(D)=\sqrt{1-G^{2}}$. O campo vetorial é então definido como:

$$
F(\mathbf{p})=v_{p}\left(G F_{\text {conv }}+H F_{\text {tang }}\right) .
$$

onde $v_{p} \equiv v_{p}\left(\tau^{*}\right)>0$ é a velocidade desejada para o robô. Neste trabalho, assumiu-se que $v_{p}$ é a velocidade da trajetória $\gamma^{i}$ avaliada no parâmetro $\tau^{*}$, assim define-se:

$$
v_{p}\left(\tau^{*}\right)=\left\|\gamma^{i}\left(\tau^{*}\right)\right\|
$$

Note que $F_{\text {conv }}$ em (11) é indefinido quando $\|\mathbf{D}\|=0$. No entanto, $D=0 \Longrightarrow G(D)=0$, assim, a componente $G F_{\text {conv }}$ em (12) corresponde ao vetor nulo quando $\|\mathbf{D}\|=0$.

3.3.2 Controle não-linear: As linhas integrais do campo vetorial (12) convergem para a curva $\gamma$. Então, o objetivo agora é fazer com que um quadrirrotor, descrito pelo modelo (1), siga este campo vetorial, de forma a impor $\dot{\mathbf{p}}=F(\mathbf{p})$.

No apêndice de Gonçalves et al. (2010), os autores apresentam um controlador apropriado para fazer o sistema integrador duplo convergir para uma linha integral de um campo vetorial. Este controlador faz o uso da matriz Jacobiana $\mathbf{J} \equiv \mathbf{J}(\overline{\mathbf{p}})$, do campo $F(\overline{\mathbf{p}})$, que é definida como:

$$
\mathbf{J}=\left[\begin{array}{llll}
\nabla F_{x} & \nabla F_{y} & \nabla F_{z}
\end{array}\right],
$$

onde $\nabla$ é o operador nabla, $F_{x}, F_{y}$ e $F_{z}$ são as componentes de $F$ em $x, y$ e $z$, respectivamente.

A lei de controle para a aceleração utilizada neste trabalho é definida com base na proposta em Gonçalves et al. (2010). Uma componente para a compensação do efeito de resistência do ar é adicionada. A lei é dada por:

$$
\mathbf{a}=\mathbf{J}^{T} \dot{\overline{\mathbf{p}}}+k_{v}(F-\dot{\overline{\mathbf{p}}})+k_{b} \frac{\dot{\overline{\mathbf{p}}}\|\dot{\overline{\mathbf{p}}}\|}{m}
$$

onde a é a aceleração de referência, $\dot{\overline{\mathbf{p}}}=R_{b w} \overline{\mathbf{v}}_{b}$ é a estimativa da velocidade do quadrirrotor no referencial inercial e $k_{v}$ é um ganho proporcional ao erro de velocidade.

Dadas as equações em (1a), (1b) e (1c), é possível calcular um valor da propulsão $u_{1}$, um valor de referência $\phi_{r}$ para a rolagem, e um valor de referência $\theta_{r}$ para a arfagem tal que $\ddot{\mathbf{p}}=\mathbf{a}$. Assim, com base na dinâmica inversa do modelo, que pode ser encontrada em Dolatabadi and Yazdanpanah (2015), define-se:

$$
\begin{aligned}
u_{1} & =\frac{m}{\cos (\bar{\phi}) \cos (\bar{\theta})}\left(g+a_{z}\right), \\
\phi_{r} & =\sin ^{-1}\left(\frac{m}{u_{1}}\left[a_{x} \sin (\bar{\psi})-a_{y} \cos (\bar{\psi})\right]\right), \\
\theta_{r} & =\sin ^{-1}\left(\frac{m}{u_{1} \cos (\bar{\phi})}\left[a_{x} \cos (\bar{\psi})+a_{y} \sin (\bar{\psi})\right]\right) .
\end{aligned}
$$

onde $a_{x}, a_{y}$ e $a_{z}$ são as componentes de $\mathbf{a}$.

A referência para a guinada é livre, assim é possível escolher uma guinada de tal forma que a câmera do drone aponte na direção da próxima janela a ser atravessada. Isso aumenta as chances do drone detectar os vértices da janela, melhorando assim a estimativa provida pelo sistema de localização. Matematicamente o ângulo de guinada de referência é definido como:

$$
\psi_{r}=\operatorname{atan} 2\left(g_{y}^{i}-\bar{y}, g_{x}^{i}-\bar{x}\right) .
$$

É ainda necessário definir leis de controle para as velocidades angulares $u_{2}, u_{3}$ e $u_{4}$. Similarmente ao que foi feito em Rezende et al. (2018), as velocidades angulares são:

$$
\begin{aligned}
& u_{2}=k_{\phi} \sin \left(\phi_{r}-\bar{\phi}\right) \\
& u_{3}=k_{\theta} \sin \left(\theta_{r}-\bar{\theta}\right) \\
& u_{4}=k_{\psi} \sin \left(\psi_{r}-\bar{\psi}\right)+\dot{\psi}_{r}
\end{aligned}
$$

A parcela de feed-forward para a guinada é definida como: 


$$
\dot{\psi}_{r}=\lim _{\delta \rightarrow 0} \frac{\sin \left[\psi_{r}(\overline{\mathbf{p}}+\delta \dot{\overline{\mathbf{p}}})-\psi_{r}(\overline{\mathbf{p}})\right]}{\delta} .
$$

Lembre-se que a razão entre $\sin (x)$ e $x$ tende a 0 quando $x \rightarrow 0$. Assim, o uso da função seno é importante em implementações práticas, uma vez atribui robustez a deslocamentos de $2 \pi$ nos ângulos envolvidos, similarmente ao que foi feito na atualização em (9). A equação em (19) é basicamente uma forma robusta de calcular a derivada temporal de $\dot{\psi}_{r}$ numericamente.

\section{Discussão}

\subsection{Replanejamento}

O planejamento de trajetória é feito com base na posição nominal das janelas, que são diferentes das posições reais. No entanto, nenhum replanejamento foi necessário. Isso se deve ao fato da estimativa da posição global ser originária da posição relativa entre o drone e a janela. Assim, uma vez que o drone assume que as janelas estão nas posições nominais, o erro de estimativa da posição do drone será igual ao deslocamento da janela em relação ao mundo.

Quando o drone passa por uma janela e começa a se localizar com base na próxima janela, a sua estimativa de posição sofrerá um deslocamento. Isso se deve ao fato das janelas possuírem deslocamentos diferentes. Neste caso, o campo vetorial fará o drone convergir novamente para a curva, ou seja, a trajetória "replanejada" corresponderá a uma linha integral do campo vetorial.

\subsection{Controlador de caminho}

O planejador utilizado neste trabalho planeja uma trajetória, não um caminho. No entanto, o controlador utilizado, baseado em campos vetoriais, foi um controlador de caminho. Note que, dado um instante $t$, a "posição desejada" do drone não está definida. O parâmetro $\tau^{*}$, na equação (10), é definido pela posição do drone, não pelo instante $t$. Assim, a referência para o controlador não é diretamente atrelada ao tempo, fazendo deste um controlador de caminho, não de trajetória.

\subsection{Implementação}

A implementação da metodologia desenvolvida neste trabalho utilizou o ROS (Robot Operating System). Diversas funções disponíveis no sistema foram exploradas, como conversão entre matrizes de rotação e ângulos de Euler. Os códigos relacionados ao planejamento de trajetória e ao estimador de estados foram todos implementados em Python. O código relacionado ao campo vetorial e ao controlador de baixo nível foi implementado em $\mathrm{C}++$. Utilizou-se a função cv2.solvePnp, da biblioteca opencv ${ }^{5}$, Python, para a execução do algoritmo PnP. No planejamento de trajetória, utilizou-se o solver de programação quadrática solvers.qp, da biblioteca cvxopt ${ }^{6}$.

\section{Resultados}

Como o trabalho consiste na união de diversas técnicas e robótica, resultados específicos de cada etapa da solução não serão o mostrados. O resultado conjunto será o foco desta seção. Para a visualização de mais resultados,

\footnotetext{
5 https://pypi.org/project/opencv-python/

6 https://pypi.org/project/cvxopt/
}

recomenda-se os vídeos em https://www. youtube.com/ channel/UCoSVIhiSbnL03jEtVumJ21g.

O ganho do campo vetorial foi $k_{f}=1.0$. Os parâmetros do planejador de trajetória foram $v_{d}=7.0 \mathrm{~m} / \mathrm{s}, d=3.0 \mathrm{~m}$ e $\delta_{q}=0.8 \mathrm{~m}$. Os ganhos associados ao controlador foram ajustados como: $k_{\phi}=k_{\theta}=3.0, k_{\psi}=0.6, k_{v}=0.6$. Os ganhos associados ao filtro foram: $K_{p}=K_{r}=0.25$ e $K_{v}=0.1$. Todos os ajustes foram feitos empiricamente no simulador FlightGoggles.

Na Figura 7 a posição nominal das janelas (previamente conhecida) é representada pelos retângulos pretos. A posição real das janelas é representada pelos retângulos amarelos. Note o deslocamento entre as posições, evidente nas janelas 13,1 e 23 . A trajetória em preto é a planejada de acordo com as posições nominais. A trajetória estimada está em vermelho. A trajetória em azul é a real executada pelo drone.

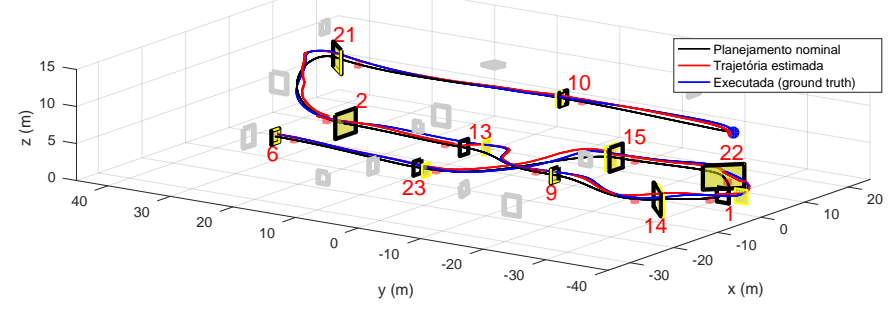

Figura 7. Trajetórias obtidas: planejada, medida e real.

A Figura 8 apresenta a vista de cima (plano xy) de um trecho do percurso da Figura 7. Nela pode-se observar como o drone corrige sua trajetória de modo a passar pela janela perturbada. Observe que a trajetória planejada (preta) passa pelas janelas nominais. Antes de atingir a janela 13, cuja posição real está muito perturbada, a estimativa da trajetória (vermelha) converge para a trajetória nominal, enquanto a posição real do drone (azul) é tal que o faz passar pela janela real (amarela). Note que a janela 9 não está perturbada com relação à pose nominal. Assim, uma vez que a janela 13 é atravessada, e o drone começa a se localizar com base na janela 9 , a trajetória estimada se aproxima da trajetória real, que juntas convergem para a trajetória nominal planejada. Outro chaveamento acontece próximo da janela 9, onde observa-se mais um deslocamento na medição de posição (em vermelho).

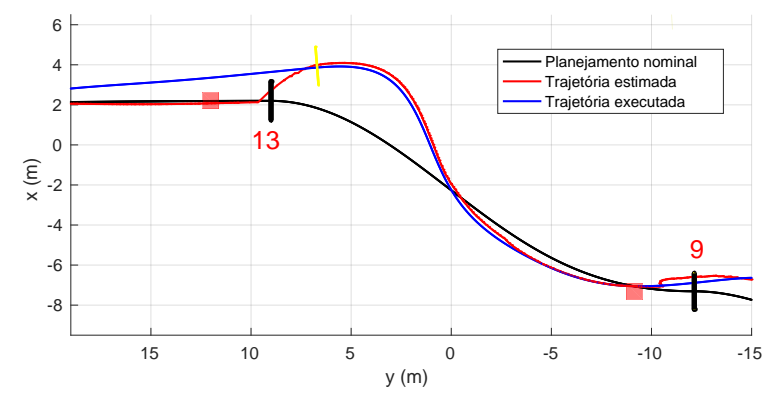

Figura 8. Exemplo de correção do percurso do drone passando pela janela 13 e indo até a janela 9 .

\section{Conclusões e trabalhos futuros}

Este trabalho apresentou a união de diversas técnicas de robótica móvel para fazer um quadrirrotor atravessar uma 
sequência de janelas. O problema resolvido é o desafio proposto pela Lookheed Martin através do AlphaPilot Challenge e foi qualificado entre as 9 melhores soluções entre um grupo de mais de 400 equipes em nível mundial.

Durante o desenvolvimento buscou-se soluções simples, eficientes e robustas. Assim, trajetórias polinomiais foram utilizadas para definir o percurso do quadrirrotor. Um observador de estados foi implementado para estimar a posição e a orientação do drone. Este filtro utiliza apenas informações da IMU e de posição relativa entre o drone e as janelas, que foi obtida a partir de um algoritmo PnP. O controle foi feito com base em campos vetoriais, que são eficientes computacionalmente e robustos.

A relativa simplicidade da solução proposta, em termos de eficiência computacional e número de sensores utilizados, é uma vantagem. De fato, esta simplicidade é um incentivo à implementação prática da estratégia. Uma necessidade seria o desenvolvimento de um algoritmo de visão computacional que vise detectar os cantos das janelas na imagem da câmera. Atualmente estas projeções são fornecidas pelo simulador FlightGoggles.

Uma dificuldade em grandes velocidades é o fato do drone apontar a câmera para baixo, o que dificulta a visualização da janela e piora a estimativa de sua pose. Um trabalho futuro importante seria a consideração de matrizes de covariância no estimador de estados. Desta forma, seria possível definir a velocidade $v_{p}$, equação (13), de forma online com base na incerteza da localização por exemplo. Assim, se a covariância da estimativa crescer, é possível diminuir $v_{p}$ para melhorar a visualização da janela e consequentemente a localização.

Como trabalhos futuros é proposto a reestruturação das equações do filtro e do controlador de forma a evitar problemas de singularidades associadas aos ângulos de Euler utilizados. Estruturas não singulares, como matrizes de rotação e quatérnions, poderão ser utilizadas. Além disso, será buscada a implementação de um sistema para prevenção de colisões. Considerando que os obstáculos são conhecidos, uma estratégia de campos vetoriais repulsivos pode ser facilmente incorporada ao controlador de campos vetoriais. Para implementar uma detecção de obstáculos online, uma possibilidade é a utilização de câmeras stereo.

\section{Agradecimentos}

Os autores agradecem aos organizadores do desafio AlphaPilot e aos colegas do VeRLab Elerson Santos, Hector Azpúrua, Maurício Ferrari, Paulo Rezeck, Renato Martins e Professor Douglas Macharet. O trabalho foi parcialmente financiado pelo Programa de Pós-Graduação em Engenharia Elétrica da UFMG (PPGEE), pela Coordenação de Aperfeiçoamento de Pessoal de Nível Superior (CAPES), pela Fundação de Amparo à Pesquisa do Estado de Minas Gerais (FAPEMIG), pelo Conselho Nacional de Desenvolvimento Científico e Tecnológico ( $\mathrm{CNPq})$, pelo Instituto Nacional de Ciência e Tecnologia para Sistemas Autônomos Cooperativos (InSAC), pelo Instituto Tecnológico Vale (ITV), pela DTI Digital, pela Fundação Christiano Ottoni (FCO), pela Escola de Engenharia da UFMG, pelo Departamento de Engenharia Elétrica da UFMG (DEE) e pelo Departamento de Ciência da Computação da UFMG (DCC).

\section{Referências}

Beard, R.W. and McLain, T.W. (2012). Small unmanned aircraft: Theory and practice. Princeton university press.

Dolatabadi, S.H. and Yazdanpanah, M.J. (2015). Mimo sliding mode and backstepping control for a quadrotor UAV. In 2015 23rd Iranian Conference on Electrical Engineering, 994-999. IEEE.

Gao, X.S., Hou, X.R., and andand Pere Hang-Fei Cheng, J.T. (2003). Complete solution classification for the perspective-three-point problem. IEEE Transactions on Pattern Analysis and Machine Intelligence, 25(8), 930943.

Gonçalves, V.M., Pimenta, L.C.A., Maia, C.A., Dutra, B.C.O., and Pereira, G.A.S. (2010). Vector fields for robot navigation along time-varying curves in ndimensions. IEEE Transactions on Robotics, 26(4), 647659.

Jayasinghe, J.A.S. and Athauda, A.M.B.G.D.A. (2016). Smooth trajectory generation algorithm for an unmanned aerial vehicle (uav) under dynamic constraints: Using a quadratic bezier curve for collision avoidance. In 2016 Manufacturing Industrial Engineering Symposium (MIES), 1-6.

Karaman, S. and Frazzoli, E. (2011). Sampling-based algorithms for optimal motion planning. The International Journal of Robotics Research, 30(7), 846-894.

Machado, H.N. and Pereira, G.A.S. (2018). Navegação de um quadrirrotor em florestas esparsas utilizando planejamento de movimento e sensor a laser. In XXII Congresso Brasileiro de Automática. João Pessoa - PB, Brasil.

Mahony, R., Kumar, V., and Corke, P. (2012). Multirotor aerial vehicles: Modeling, estimation, and control of quadrotor. IEEE Robotics Automation Magazine, 19(3), 20-32.

Mellinger, D. and Kumar, V. (2011). Minimum snap trajectory generation and control for quadrotors. In 2011 IEEE International Conference on Robotics and Automation, 2520-2525.

Rezende, A.M.C., Gonçalves, V.M., Raffo, G.V., and Pimenta, L.C.A. (2018). Robust fixed-wing UAV guidance with circulating artificial vector fields. In 2018 IEEE/RSJ International Conference on Intelligent Robots and Systems (IROS), 5892-5899.

Richter, C., Bry, A., and Roy, N. (2016). Polynomial trajectory planning for aggressive quadrotor flight in dense indoor environments. In Robotics Research, 649666. Springer.

Sayre-McCord, T., Guerra, W., Antonini, A., Arneberg, J., Brown, A., Cavalheiro, G., Fang, Y., Gorodetsky, A., McCoy, D., Quilter, S., Riether, F., Tal, E., Terzioglu, Y., Carlone, L., and Karaman, S. (2018). Visual-inertial navigation algorithm development using photorealistic camera simulation in the loop. In 2018 IEEE International Conference on Robotics and Automation (ICRA).

Thrun, S., Burgard, W., and Fox, D. (2005). Probabilistic robotics. MIT press.

Yousefi, F. and Monfared, S.B. (2018). Cascade feedback linearization controller with actuators dynamic for trajectory tracking of flying robot. In 2018 8th Conference of AI Robotics and 10th RoboCup Iranopen International Symposium (IRANOPEN), 46-51. 